\title{
Management of Gut Microbiota to Improve Sleep Dysfunction for Children with Autism-Spectrum Disorders
}

\author{
Vasanth Senthilraja ${ }^{1}$, Ethan Yang ${ }^{1}$, Akshay Jakkidi Reddy ${ }^{1}$, Eric Liu ${ }^{2}$ and Himanshu Wagh ${ }^{3 *}$ \\ ${ }^{1}$ California Northstate University College of Health Sciences, Rancho Cordova, USA \\ ${ }^{2}$ University of California Los Angeles, Los Angeles, USA \\ ${ }^{3}$ California Northstate University College of Medicine, Elk Grove, USA
}

*Corresponding author: Himanshu Wagh, California Northstate University College of Medicine, Elk Grove, USA, Email: Himanshu.wagh5630@cnsu.edu

\section{ABSTRACT}

Many children with Autism-Spectrum Disorders (ASD) struggle with sleep dysfunction. This can be caused by a lack of important gut microbiota (GM) that have the ability to influence functions of the nervous system through the gut-brain axis. The metabolites of GM function are responsible for influencing the production of pertinent sleep hormones, such as melatonin and serotonin. The research has identified lower abundance levels of the gut microbiota species Faecalibacterium and Agathobacter in children with ASD that struggled with sleep disorders. When exploring their impact on sleep hormone production, a positive correlation was identified between these species of GM and melatonin levels, which regulates circadian sleep cycles. In addition, a negative correlation was observed between these species of GM and serotonin levels, which high levels of can lead to wakefulness and sleep dysfunction. To improve GM levels in children with ASD, a Bimuno ${ }^{\circledR}$ galactooligosaccharide (B-GOS $\left.{ }^{\circledR}\right)$ prebiotic intervention was tested in children with ASD. While this intervention led to improved GM levels and increased levels of Faecalibacterium growth, a significant difference was not noticed between groups. Furthermore, an analysis of probiotic intervention studies in various individuals revealed the ability to improve sleep metric scores through consumption of probiotics. These findings can be further explored in children with ASD for potential treatments for sleep disorders.

\section{INTRODUCTION}

The human gut microbiota can influence physiological functions through the body's gut-brain axis (GBA). The GBA consists of a connection between the central and the enteric nervous system (NS), linking neurological functions of the brain with peripheral intestinal functions [1]. It is currently understood that the GBA is heavily influenced by the gut microbiome [2]. The GBA has been known to exhibit bidirectional signaling, where not only can gut bacteria influence health and behavior, but neurological states can alter gut health [2]. As such, any changes in the nervous
Keywords

Autism-spectrum disorders

Gut-brain axis

Gut microbiota

Melatonin

Serotonin system, such as increased stress levels, or sleep dysfunction, also affects the GBA [2]. The bottom-up control of the NS by the body's microbiome primarily occurs through neuroimmune and neuroendocrine mechanisms, often involving the vagus nerve [3]. Studies have found that vagal nerve stimulation can lead to improvement in general autism symptoms, such as sleep, eating, and social interactions in children, by antiinflammatory effects that modulate production of TNF- $\alpha$ and IL-6 cytokines [4]. Within the microbiome, the short chain fatty acids (SCFA, e.g. butyrate, acetate) produced by fermenting 


\section{Research Article}

bacteria can suppress pro-inflammatory cytokines and interact with regulatory $\mathrm{T}$ cells to attenuate colitis [1]. This shows how the gut microbiome can influence overall physiological functions in the body through the GBA.

Autism-spectrum disorder (ASD) is a type of neurodevelopmental disease characterized by impaired social interactions and communication, accompanied by stereotypic behavior, and restricted interests or activities [5]. Those who suffer from ASD have been known to have issues with sleeping, which as shown, can further exacerbate the symptoms of ASD that these individuals already have [5]. The role gut microbiota (GM) play in influencing sleep function through the GBA can be utilized to understand sleep dysfunction in individuals with ASD. Studies show that around $80 \%$ of children with ASD struggle with sleep problems [6]. The microbiome can be affected using prebiotics and probiotics. Prebiotics are a source of nutrients for the microbes in the microbiome [7]. These are the carbohydrates that cannot be digested by the body naturally. The use of prebiotic interventions has shown promising results for improving GM diversity and cultivating the growth of specific GM that affect sleep function [8]. Compared to prebiotics, probiotics are living microbes that are beneficial to the body [9]. Like prebiotics, probiotic intervention has also led to improvements in sleep function in children with ASD [9].

The role gut microbiota plays in impacting sleep function occurs through regulation of circadian rhythms of the host [10]. Gut microbial metabolites influence central and hepatic clock gene expression and sleep duration in the host and regulate body composition through circadian transcription factors [11]. Circadian rhythms are regulated by intracellular clocks composed of interacting genes and proteins that facilitate daily oscillations in gene expression [12]. Microbederived metabolites such as short-chain fatty acids and bile acids are likely mediators for gut microbiota-host circadian communication [13]. For example, dietary choline is converted to trimethylamine by the intestinal microbiota and is converted to trimethylamine N-oxide (TMAO) in the liver, which influence the expression of circadian clock genes (including Clock and Bmall) in endothelial cells [14]. Within this axis, the microbiota in the gut affect brain function through 3 pathways that produce a bidirectional flow of information, as seen in Figure 1 [15]. The first of these is the immunoregulatory pathway, in which the microbiota interact with immune cells in such a way as to affect the levels of cytokines, cytokinetic reaction factor, and prostaglandin E2 [16]. The second is the neuroendocrine pathway that contains more than 20 types of enteroendocrine cells in the intestine [17]. The third is the vagus nerve pathway, in which the enteric nervous system plays an important role [18]. Anatomical evidence indicates that the sensory neurons of the intestinal myenteric plexus are exposed to the gut microbiota; these neurons form synaptic contacts with motor neurons in the intestine that are involved in the regulation of intestinal motility and gut hormone secretion [18]. Whether gut microbiota rhythmicity is regulated by endogenous bacterial clocks, or is simply a reflection of the community responding to host circadian processes is unclear.

The purpose of this literature review is to study how variations in GM diversity affects the functioning of sleep in children with ASD. The research study aims to identify specific microbial species that have an impact on hormones, such as melatonin, that influence sleep function. By identifying specific microbiota that influence the production of sleep hormones, prebiotic and probiotic interventions can be used to foster the growth of such GM to improve sleep function. These types of interventions will also be studied to understand their potential clinical impact for treating sleep disorders in children with ASD.

\section{METHODOLOGY}

A wide search was performed across research journals and online sources using keywords "Autism-Spectrum Disorder", "sleep disorder", "Gut-Brain Axis", "probiotics", "probiotics", and "microbes". Potential research studies were collected and analyzed from online databases such as PubMed, NCBI, and Science Daily. The research studies that contained concrete scores for evaluating ASD and sleep disorders were selected and the data from these studies were compiled into an excel sheet. A variety of sleep score metrics, such as the Modified Simonds \& Parraga Sleep Questionnaire (MSPSQ), Pittsburgh Sleep Quality Index (PSQI), and the Children's Sleep Habits Questionnaire (CSHQ), were compared for effectiveness of measuring sleep quality. The studies that lacked improvements in sleep scores and did not explain the specific microbiota identified to influence sleep were discarded. Sources related to the gut-brain axis yielded information that explained the role microbiota play in influencing metabolite production that impacts sleep disorders in people with ASD. This information was filtered for selective microbes and their direct effects on specific hormones and metabolites that influence sleep physiology. The correlation between sleep quality scores and specific microbiota abundance levels was calculated and analyzed using a Spearman's Rank Correlation Coefficient $\left(\mathrm{R}_{\mathrm{s}}\right)$. The statistical significance of the measured correlation was determined using a calculated $p$-value, with a $p<0.005$ indicating a significant difference between analyzed groups. A meta-analysis was conducted to further analyze and filter 


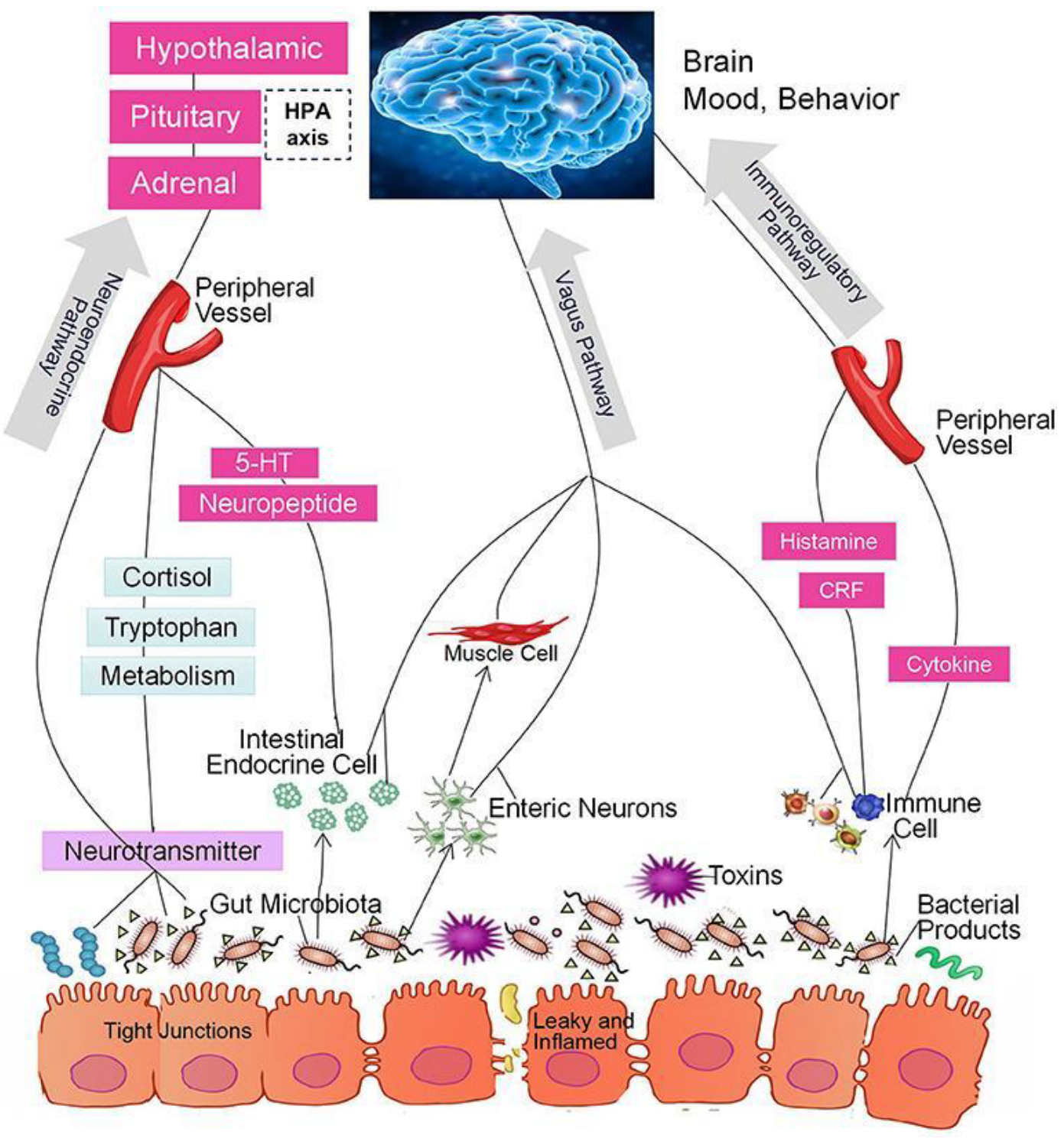

Figure 1: Overview of how gut microbiota affect the gut-brain axis through immunoregulatory, neuroendocrine, and vagus pathways [15].

the studies based on a common measurement parameter for interventions that affected the microbiotas that were identified to play a role in influencing sleep function. A search was conducted for studies that discussed probiotic treatment options for improving sleep disorders. The literature sources related to treatment and prebiotics helped discover potential intervention studies that improve sleep scores for people with ASD through microbiota management. The results of the search were compiled based on a common sleep metric and listed as data in the study. The data was analyzed based on parameters, such as the study participants, the specific microbiota introduced through probiotics, the colony factor units (CFU), the duration of the intervention, and the measured sleep quality scores.

\section{DATA AND RESULTS}

Table 1: Correlation analysis between microbiota abundance levels and ASD symptom scores [19].

\begin{tabular}{|l|c|c|c|c|}
\hline & Faecalibacterium & & Agathobacter & \\
\hline & $\mathrm{R}_{\mathrm{s}}$ & $\mathrm{p}$-value & $\mathrm{R}_{\mathrm{s}}$ & $\mathrm{p}$-value \\
\hline CHSQ & -0.244 & $0.007^{*}$ & -0.225 & $0.014^{*}$ \\
\hline CARS & -0.018 & 0.844 & -0.046 & 0.616 \\
\hline
\end{tabular}

Table 2: Correlation analysis between microbiota abundance levels and metabolites [19].

\begin{tabular}{|l|c|c|c|c|}
\hline & Faecalibacterium & & Agathobacter & \\
\hline & $\mathrm{R}_{\mathrm{s}}$ & $\mathrm{p}$-value & $\mathrm{R}_{\mathrm{s}}$ & $\mathrm{p}$-value \\
\hline Serotonin & -0.018 & 0.844 & -0.046 & 0.616 \\
\hline Melatonin & 0.197 & $0.036^{*}$ & 0.192 & $0.041^{*}$ \\
\hline
\end{tabular}


Table 3: Comparison of probiotic intervention studies and their effects on sleep scores for various participants.

\begin{tabular}{|c|c|c|c|c|c|c|}
\hline Citation & Participants & $\begin{array}{l}\text { Intervention } \\
\text { Treatment }\end{array}$ & Probiotic Bacteria & CFU & Duration & $\begin{array}{l}\text { Mean } \Delta \\
\text { PSQI }\end{array}$ \\
\hline Dhiman [20] & $\begin{array}{l}\text { PLA: } 11 \text { PRO: } \\
16\end{array}$ & $\begin{array}{l}1 \text { x VSL\#3 } \\
\text { Sachet Daily }\end{array}$ & $\begin{array}{l}\text { L. casei, L. plantarum, L. acidophilus, } \\
\text { L. delbrueckii bulgaricus, B. longum, } \\
\text { B. breve, B. infantis, Streptococcus } \\
\text { thermophilus }\end{array}$ & $9 \times 10^{\wedge} 11$ & 24 weeks & 1.32 \\
\hline Kato [21) & $\begin{array}{l}\text { PLA: } 23(12 \mathrm{M}) \\
\text { PRO: } 24(14 \mathrm{M})\end{array}$ & $\begin{array}{l}100 \mathrm{ml} \\
\text { Fermented Milk } \\
\text { Daily }\end{array}$ & L. casei shirota & $1 \times 10 / \mathrm{ml}$ & 8 weeks & 1.20 \\
\hline Marotta [22] & $\begin{array}{l}\text { PLA: } 15(10 \mathrm{M}) \\
\text { PRO: } 18(11 \mathrm{M})\end{array}$ & 1 x Sachet Daily & $\begin{array}{l}\text { L. fermentum, L. rhamnosus, L. } \\
\text { plantarum, B. longum }\end{array}$ & $4 \times 10^{\wedge} 9$ & 6 weeks & 1.27 \\
\hline Nishida [23] & $\begin{array}{l}\text { PLA: } 16(10 \mathrm{M}) \\
\text { PRO: } 16(11 \mathrm{M})\end{array}$ & $\begin{array}{l}200 \mathrm{ml} \\
\text { Fermented Milk } \\
\text { Dailyt }\end{array}$ & heat-inactivated L. gasseri & $1 \times 10^{\wedge} 10$ & 5 weeks & 1.76 \\
\hline Sawada [24] & $24 \mathrm{M}$ & $1 \times$ Sachet Daily & L. gasseri & $1 \times 10^{\wedge} 10$ & 4 weeks & 1.69 \\
\hline
\end{tabular}
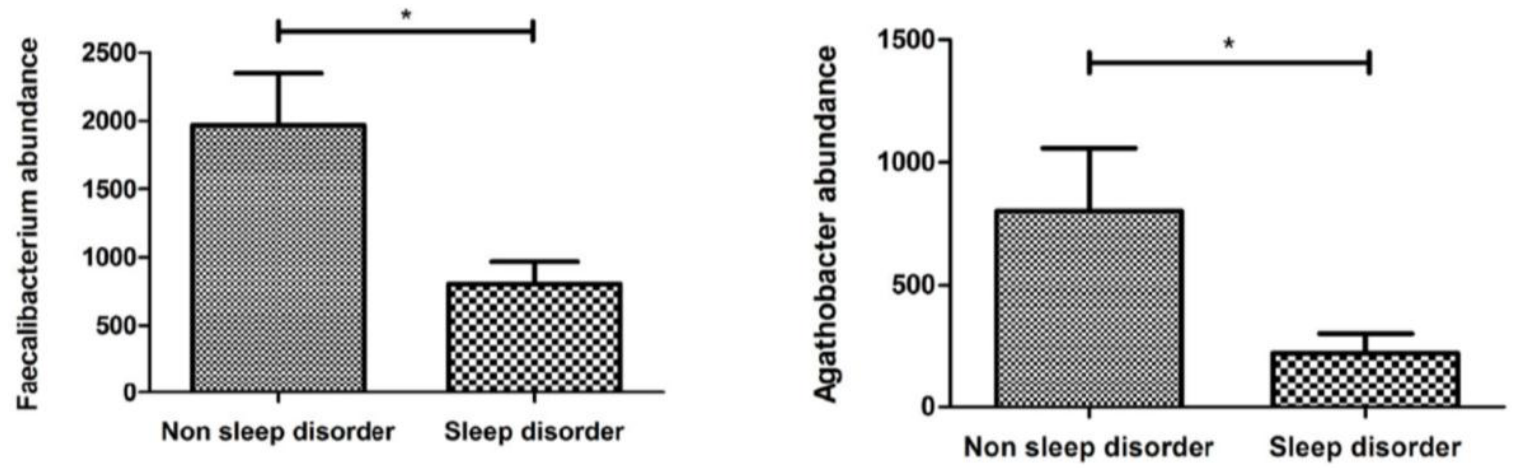

Figure 2: Differences in Faecalibacterium and Agathobacter abundance between individuals with sleep disorders and non-sleep disorder [19].

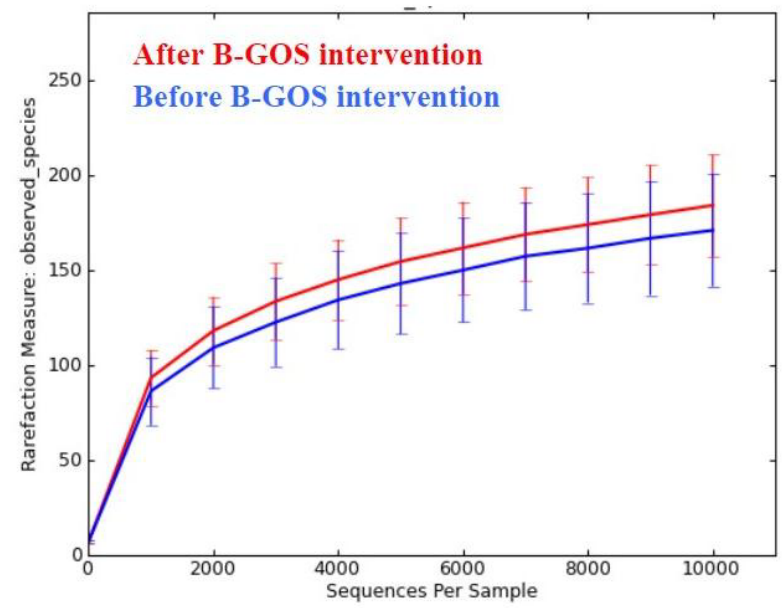

Figure 3: Effects of B-GOS ${ }^{\circ}$ prebiotic intervention on microbiota growth [8].

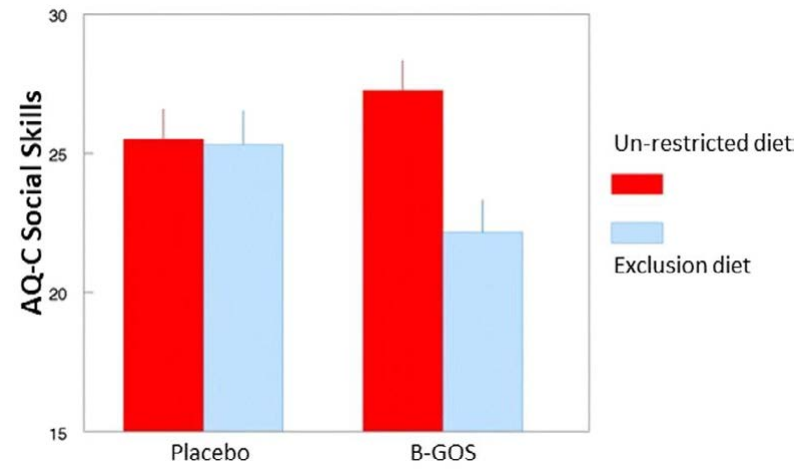

Figure 4: Comparison of B-GOS and placebo effect on AQ-C scores for children with autism [8]. 


\section{RESULTS}

The analysis of stool samples collected from children with ASD revealed an abundance of Faecalibacterium and Agathobacter among various other microbes. These children were evaluated for having a sleep disorder using a Children Sleep Habits Questionnaire (CSHQ) score [19]. The severity of their ASD condition was evaluated using a Childhood Autism Rating Scale (CARS) score. The abundance levels of Faecalibacterium and Agathobacter were compared between ASD children with and without sleep disorders. The comparison revealed that children with sleep disorders had lower abundance levels of Faecalibacterium and Agathobacter in their stool samples compared to children without a sleep disorder. A negative correlation was identified between the CHSQ scores and Faecalibacterium and Agathobacter abundance levels, with a $\mathrm{p}>0.005$ for both bacteria, indicating a significant difference between the two ASD test groups. Similarly, a negative correlation existed between CARS scores and Faecalibacterium and Agathobacter abundance levels. The impact of these microbiota on metabolites that influence sleep disorders were also analyzed by measuring serotonin and melatonin levels in both test groups. A positive correlation was measured between melatonin levels and Faecalibacterium and Agathobacter abundance levels. A negative correlation was measured between serotonin levels and Faecalibacterium and Agathobacter abundance levels.

An intervention study in children with ASD that involved the application of prebiotic Bimuno ${ }^{\circledR}$ galactooligosaccharide (B-GOS $\left.{ }^{\circledR}\right)$ alongside exclusion diets was analyzed to test for the effect of GM manipulation on autism symptoms. The testing showed that B-GOS $₫$ intervention led to an increase in microbiota levels in test subjects with a $\mathrm{p}<0.004$. Compared to the placebo, the subjects that were given a B-GOS ${ }^{\circledR}$ exclusion diet with modifications of different nutrients reported lower autism spectrum quotient (AQ-C) scores, whereas a B-GOS ${ }^{\circledR}$ unrestricted diet led to worsening of AQ-C scores. A qualitative analysis that involved test subjects keeping sleep diaries showed that $23 \%$ of participants (two ASD following unrestricted diet and one under exclusion diet) benefited from B-GOS ${ }^{\circledR}$ intervention [8]. The parents reported their children had less problems falling asleep and slept $1 \mathrm{~h}$ longer than usual [8].

The literature review found several probiotic interventions studies that modified specific GM across a range of participants and revealed increases in Pittsburgh Sleep Quality Index (PSQI) scores. Of the five studies analyzed, the first four studies followed a between subject's parallel design while the last study followed a within subject's crossover design. The information regarding the intervention treatment and specific microbe targeted was reported along with the colony forming unit (CFU) and duration of the treatment. None of the participants in these studies, however, were reported of having ASD. The number of individuals receiving the placebo treatment group (PLA) and probiotics treatment group (PRO) were also reported as data. The change in PSQI scores between the PLA and PRO groups was measured and recorded as Mean $\triangle$ PSQI data, with greater change values indicating improved efficacy of intervention treatment.

\section{DISCUSSION}

The presence of specific GM, such as Faecalibacterium and Agathobacter, influences sleep physiology in children with ASD. Based on CHSQ scores in Table 1, children reported to have sleep disorders were found with lower Faecalibacterium and Agathobacter abundance levels. The negative correlation between Faecalibacterium and Agathobacter abundance levels and CHSQ scores indicates that the lack of these microbiota can lead to sleep dysfunction in children with autism. Furthermore, the negative correlation between Faecalibacterium and Agathobacter abundance levels and CARS scores in Table 1 shows that the lack ofGMcaninfluence the onset of general autism symptoms. The analysis of specific metabolites in the children with ASD helped demonstrate how GM impact sleep function. As a positive correlation existed between Faecalibacterium and Agathobacter abundance levels and melatonin levels, the ASD children with lower CHSQ scores struggled with sleep disorders due to a lack of melatonin produced. As melatonin plays a role in regulating circadian rhythms, the lower melatonin levels can be linked to sleep dysfunction in children with ASD [25]. However, there was an increase in serotonin levels in the sleep disorder group. As certain levels of serotonin can contribute more towards wakefulness than sleep, the negative correlation between serotonin levels and microbiota can be attributed to sleep dysfunction [26]. Overall, the correlations between certain GMa and sleep-inducing metabolites suggests that children with ASD struggle with sleep disorders due to an imbalance of important sleep hormones generated by GM. The $\mathrm{p}>0.005$ affirms that these findings are significant differences between the ASD groups with sleep disorders and without sleep disorders.

The use of prebiotic intervention shows promising results for improving sleep disorders and other symptoms of ASD in children. While the study examined was carried out for the sake of improving GI discomfort in children with autism, the results show other promising findings [8]. Based on Figure 3, children with ASD showed an increase in diversity of GM composition after receiving B-GOS ${ }^{\circledR}$ prebiotics. However, the $p<0.004$ for the data indicates that there was not a significant difference 
between the groups. The analysis of the different microbiota species revealed higher presence of Faecalibacterium prausnitzii in samples, the GM associated with improved CHSQ scores [8]. When examining AQ-C scores, which encompassed a range of autism symptoms including sleep function, the children that were on an exclusion diet with $\mathrm{B}-\mathrm{GOS} \AA$ prebiotics exhibited a significant reduction in AQ-C scores, as seen in Figure 4. The increase in AQ-C scores associated with an unrestricted diet with $B-G O S \circledR$ prebiotics indicates the need for diet regulation along with prebiotic intervention. The qualitative analysis based on sleep diaries also showed improvements in sleep for children with ASD that received the exclusion diet with B-GOS ${ }^{\circledR}$ prebiotics [8]. However, a significant difference was not found between groups due to difficulties in consistent reports and lack of proper evaluation tools. Overall, such prebiotic intervention studies can be further revised and explored for improving GM that can lead to improvements in sleep disorder conditions for children with ASD.

The mean change reductions in PSQI scores across probiotic interventions for sleep disorders suggest that consumption of certain probiotics can improve sleep quality. The studies in Table 3 reported a mean pretreatment score of 5.59, with a range of 4.57 to 6.63 PSQI scores. According to the PSQI, a global score greater than 5 indicates poor sleep quality [27]. Hence, the mean change in PSQI scores for some of these studies does show clinical improvement in sleep quality. One of the studies reported positive correlations between the measured PSQI scores and features of mood, such as anxiety and depressive symptoms [23]. However, since PSQI scores are generated using subjective responses, the need of further testing through EEG or actigraphy is needed to confirm these results. Like other self-reported inventories, PSQI scores are subject to exaggeration by study participants, leading to lower accuracy in results [27]. Nonetheless, since PSQI scores are a known and valid assessment of sleep quality, the change in PSQI scores does exhibit a clinical significance in improving sleep quality through probiotic intervention [27]. The impact of these supplements is still small and requires further testing with other metrics for significant treatment outcomes. Also, the fact that the participants in the studies did not have ASD limits the generalization of the findings for children with ASD. However, future research can aim towards regulated randomized testing of such probiotic interventions in children with ASD to study improvements in sleep dysfunction.

\section{CONCLUSION}

The findings of the research study reveal a connection between gut microbiota and onset of autism symptoms, such as sleep dysfunction. The lack of certain microbiota, such as Faecalibacterium and Agathobacter, are associated with higher CHSQ scores that indicate the onset of sleep disorders. Furthermore, the impact of these GM on important sleep hormones, such as melatonin and serotonin, and their levels reveals how the gut-brain axis influences sleep disorders. The use of prebiotic and probiotic interventions shows promising treatment options for improving sleep dysfunction in children with ASD. The B-GOS ${ }^{\circledR}$ prebiotic intervention with an exclusion diet led to reduction in AQ-C scores that highlighted a range of autism symptoms. However, the prebiotic intervention study did not have a statistically significant difference between test groups and the probiotic interventions were not tested out in children with ASD. While the findings from probiotic intervention cannot be generalized to children with ASD, further research can be carried to test out such treatments in children with ASD for improvements in sleep functions.

\section{REFERENCES}

1. Carabotti M, Scirocco A, Maselli MA, Severi C (2015) The gutbrain axis: interactions between enteric microbiota, central and enteric nervous systems. Annals of gastroenterology, 28(2): 203209.

2. Smith RP, Easson C, Lyle SM, Kapoor R, Donnelly CP, et al. (2019) Gut microbiome diversity is associated with sleep physiology in humans. PLOS ONE, 14(10). https://doi.org/10.1371/journal. pone. 0222394

3. Smith RP, Easson C, Lyle SM, Kapoor R, Donnelly CP, et al. (2019) Gut microbiome diversity is associated with sleep physiology in humans. PLOS ONE, 14(10). https://doi.org/10.1371/journal. pone. 0222394

4. Derakhshan N (2015) Vagal nerve stimulation for the treatment of autism. Mental Illness, 7(1). https://doi.org/10.4081/mi.2015.5788

5. Devnani PA, Hegde AU (2015) Autism and sleep disorders. Journal of Pediatric Neurosciences, 10(4): 304. https://doi. org/10.4103/1817-1745.174438

6. Mazzone L, Postorino V, Siracusano M, Riccioni A, Curatolo P (2018) The relationship between sleep problems, neurobiological alterations, core symptoms of autism spectrum disorder, and psychiatric comorbidities. Journal of Clinical Medicine, 7(5): 102. https://doi.org/10.3390/jcm7050102

7. Holscher HD (2017) Dietary fiber and prebiotics and the gastrointestinal microbiota. Gut Microbes, 8(2): 172-184. https:// doi.org/10.1080/19490976.2017.1290756

8. Grimaldi R, Gibson GR, Vulevic J, Giallourou N, Castro-Mejía JL, Hansen LH, Leigh Gibson E, Nielsen DS, Costabile A (2018) A prebiotic intervention study in children with autism spectrum disorders (ASDS). Microbiome, 6(1). https://doi.org/10.1186/ s40168-018-0523-3

9. Mitchell LK, Davies PS (2021) Pre- and probiotics in the management of children with autism and gut issues: A review of the current evidence. European Journal of Clinical Nutrition. https://doi.org/10.1038/s41430-021-01027-9 
10. Parkar S, Kalsbeek A, Cheeseman J (2019) Potential role for the gut microbiota in modulating host circadian rhythms and Metabolic Health. Microorganisms, 7(2): 41. https://doi. org/10.3390/microorganisms 7020041

11. Li Q, Han Y, Dy A, Hagerman R (2017) The gut microbiota and autism spectrum disorders. Frontiers in Cellular Neuroscience, 11. https://doi.org/10.3389/fncel.2017.00120

12. Marcheva B, Ramsey KM, Buhr ED, Kobayashi Y, Su H, Ko CH, Ivanova G, Omura C, Mo S, Vitaterna MH, Lopez JP, Philipson LH, Bradfield CA, Crosby SD, JeBailey L, Wang X, Takahashi JS, Bass J (2010) Disruption of the clock components clock and BMAL1 leads to hypoinsulinaemia and diabetes. Nature, 466 (7306): 627-631. https://doi.org/10.1038/nature09253

13. Frazier K, Chang EB (2020) Intersection of the gut microbiome and circadian rhythms in metabolism. Trends in Endocrinology \& Metabolism, 31(1): 25-36. https://doi.org/10.1016/j. tem.2019.08.013

14. Bishehsari F, Voigt RM, Keshavarzian A (2020) Circadian rhythms and the gut microbiota: From the metabolic syndrome to cancer. Nature Reviews Endocrinology, 16(12): 731-739. https:// doi.org/10.1038/s41574-020-00427-4

15. Li Y, Hao Y, Fan F, Zhang B (2018) The role of microbiome in insomnia, circadian disturbance and depression. Frontiers in Psychiatry, 9. https://doi.org/10.3389/fpsyt.2018.00669

16. Feng Q, Chen, WD, Wang, YD (2018) Gut microbiota: An integral moderator in health and disease. Frontiers in Microbiology, 9. https://doi.org/10.3389/fmicb.2018.00151

17. Raybould HE (2010) Gut chemosensing: Interactions between gut endocrine cells and visceral afferents. Autonomic Neuroscience, 153(1-2): 41-46. https://doi.org/10.1016/j.autneu.2009.07.007

18. Powley T, Wang X, Fox E,Phillips R, Liu L, et al. (2007) Ultrastructural evidence for communication between intramuscular vagal mechanoreceptors and interstitial cells of cajal in the rat fundus. Neurogastroenterology \& Motility. https:// doi.org/10.1111/j.1365-2982.2007.00990.x

19. Hua X, Zhu J, Yang T, Guo M, Li Q, et al. (2020) The gut microbiota and associated metabolites are altered in sleep disorder of children with autism spectrum disorders. Frontiers in Psychiatry, 11. https://doi.org/10.3389/fpsyt.2020.00855
20. Dhiman RK, Rana B, Agrawal S, Garg A, Chopra M, et al. (2014) Probiotic VSL\#3 reduces liver disease severity and hospitalization in patients with cirrhosis: A randomized, controlled trial. Gastroenterology, 147(6). https://doi.org/10.1053/j. gastro.2014.08.031

21. Kato-Kataoka A, Nishida K, Takada M, Suda K, Kawai M, Shimizu K, Kushiro A, Hoshi R, Watanabe O, Igarashi T, Miyazaki K, Kuwano Y, Rokutan K (2016) Fermented milk containing lactobacillus casei strain shirota prevents the onset of physical symptoms in medical students under academic examination stress. Beneficial Microbes, 7(2): 153-156. https:// doi.org/10.3920/bm2015.0100

22. Marotta A, Sarno E, Del Casale A, Pane M, Mogna L, et al. (2019) Effects of probiotics on cognitive reactivity, mood, and sleep quality. Frontiers in Psychiatry, 10. https://doi.org/10.3389/ fpsyt.2019.00164

23. Nishida K, Sawada D, Kawai T, Kuwano Y, Fujiwara S, Rokutan K (2017) Para-psychobiotic lactobacillus gasseri CP 2305 ameliorates stress-related symptoms and sleep quality. Journal of Applied Microbiology, 123(6): 1561-1570. https://doi. org/10.1111/jam.13594

24. Sawada D, Kawai T, Nishida K, Kuwano Y, Fujiwara S, Rokutan K (2017) Daily Intake of Lactobacillus gasseri CP2305 improves mental, physical, and sleep quality among Japanese medical students enrolled in a cadaver dissection course. Journal of Functional Foods, 31, 188-197. https://doi.org/10.1016/j. jff.2017.01.042

25. Wu, Z.-yue, Huang, S.-dai, Zou, J.-jun, Wang, Q.-xin, Naveed M, Bao, H.-nan, Wang W, Fukunaga K, Han F (2020) Autism spectrum disorder (ASD): Disturbance of the melatonin system and its implications. Biomedicine \& Pharmacotherapy, 130, 110496. https://doi.org/10.1016/j.biopha.2020.110496

26. Kheirouri S, Kalejahi P, Noorazar SG (2016) Plasma levels of serotonin, gastrointestinal symptoms, and sleep problems in children with autism. Turkish Journal of Medical Sciences, 46, 1765-1772. https://doi.org/10.3906/sag-1507-68

27. Grandner MA, Kripke DF, Yoon IY, Youngstedt SD (2006)Criterion validity of the Pittsburgh Sleep Quality index: Investigation in a non-clinical sample. Sleep and Biological Rhythms, 4(2): 129136. https://doi.org/10.1111/j.1479-8425.2006.00207.x 